\title{
Aspectos epidemiológicos da tuberculose multirresistente em serviço de referência na cidade de São Paulo
}

\author{
Epidemiological features of multidrug-resistant tuberculosis \\ in a reference service in São Paulo city
}
Fernando Augusto Fiuza de Melo', Jorge Barros Afiune1, Jorge Ide Neto', Elisabete Aparecida de Almeida', Delurce Tadeu Araujo Spada', Augusta Nunes Lemos Antelmo ${ }^{1}$ e Maria Luiza Cruz ${ }^{1}$

\begin{abstract}
Resumo Com o objetivo de estudar algumas características epidemiológicas dos portadores de tuberculose pulmonar multirresistente e suas influências sobre o controle e o tratamento, foi avaliada uma coorte de 4 anos de pacientes selecionados pela recuperação do Mycobacterium tuberculosis no escarro, resistência à rifampicina, isoniazida e mais uma terceira droga usual ou falência do esquema de reserva, matriculados em uma referência na cidade de São Paulo. As variáveis estudadas foram: sexo e idade, tipo de multirresistência, contágio, condições associadas, perfil de resistência às drogas usuais e distribuição das lesões na radiologia convencional. Revistos 182 pacientes, $112(61,5 \%)$ masculinos, com idade variando entre 16 e 64 anos $(35,7 \pm 6,8)$. Com base na história terapêutica foram discriminados os seguintes tipos: MR-primária (com teste de sensibilidade inicial), 11 (6\%), MR-pós-primária (irregularidade no tratamento anterior), 134 (74\%) e MR-indeterminada (falência após uso regular informado dos esquemas usuais), 37 (20\%). Contágio presente em 41 de 170 pacientes, predominando o intradomiciliar sobre o institucional. Identificados 4 surtos familiares e nenhum institucional. $O$ abandono (45\%) foi a mais freqüente condição associada, seguido do etilismo (27\%), falência seqüencial aos esquemas de retratamento (23\%), contágio com multirresistência (15\%), reações adversas às drogas (6\%), HIV-positivo (4\%) e diabetes (3\%). Resistência à rifampiina+isoniazida em 100\%, 83\% à estreptomicina e $47 \%$ ao etambutol. Todos com cavidades no Rx de tórax convencional, unilaterais em 35 (19\%). Discutem-se os achados e apresentam-se sugestões.
\end{abstract}

Palavras-chaves: Tuberculose. Tuberculose multirresistente. Controle e tratamento da multirresistência.

Abstract In order to study certain epidemiological features of multidrug-resistant tuberculosis (MDR-TB) carriers and their influence on the control and treatment, a group of patients was evaluated over a four-year period, selected by: Mycobacterium tuberculosis isolation from sputum; resistance to Rifampin, Isoniazid and one more drug, or, failure of reserve regimen, all cases were from a tuberculosis reference unit in the City of São Paulo. A total of 182 patients were reviewed, with a mean age of $35.7 \pm 6.8$ years and $112(61.5 \%)$ were male. These patients was classified according to therapeutic history, as: primary MDR-TB (with initial sensitivity test) 11 (6\%); post primary MDR-TB (after irregular use previous treatment) $134(74 \%)$, and indeterminate MDR-TB (failure after regular use of initial and reserve regimens) 37 (20\%). Contagion was identified in 41/170 patients, acquired through domiciliary rather than institutional transmission. There were four familial outbreaks and none were institutional. The most frequent condition associated with these cases was abandonment of therapy (45\%) followed by alcoholism (27\%), sequential failure in the treatment regimens (23\%), MDR contagion (15\%), drug reaction (6\%), HIV positive (4\%) and diabetes (3\%). There was resistance to Rifampin+lsoniazid in $100 \%$, Streptomycin in $83 \%$ and Ethambutol in $47 \%$. Conventional $X$-ray revealed cavities in all, though only 35 (19\%) were unilateral. These cases are discussed and some suggestions presented.

Key-words: Tuberculosis. Multidrug-resistant tuberculosis. Control and treatment of MDR-TB.

\footnotetext{
1. Divisão de Tisiologia e Pneumologia Sanitária do Instituto Clemente Ferreira da Secretaria de Estado da Saúde de São Paulo, São Paulo, SP, Brasil. Endereço para correspondência: Dr. Fernando Augusto Fiuza de Melo. Divisão de Tisiologia e Pneumologia Sanitária/Instituto Clemente Ferreira/ SES-SP. R. da Consolação 717, Consolação, 01301-000 São Paulo, SP.

Telefax: 5511 3257-8096/3258-7644

e-mail:fernandofiuza@terra.com.br

Recebido em 10/10/2001.
} 
Entre os problemas da tuberculose (TB) recrudescente nos países avançados, permanecente e agravada nos países em desenvolvimento, pelo aumento da miséria, coinfecção com o HIV/AIDS, deterioração dos serviços de saúde e progressiva redução de recursos assistenciais, um dos mais preocupantes e discutidos tem sido o fenômeno da multirresistência (MR) 272831 .

A tuberculose multirresistente (TBMR), que vem sendo definida na literatura internacional como uma doença provocada por cepas do Mycobacterium tuberculosis resistentes a mais de uma droga, em especial à rifampicina $(\mathrm{R})$ e à isoniazida $(\mathrm{H})$, dupla de maior potencial bactericida e esterilizante no tratamento da doença, preocupa, seja pela possibilidade de disseminação de cepas multirresistentes (MR), como pelas dificuldades de se estabelecer esquemas terapêuticos eficazes e efetivos ${ }^{29} 32$.

No Brasil, o Ministério da Saúde (MS) adota normativamente, dois diferentes esquemas terapêuticos para a TB. Inicialmente, o Esquema-1 (E-1), com R e H por seis e pirazinamida (Z) nos dois primeiros meses, é indicado para pacientes sem tratamento anterior ou para aqueles que recidivam após cura ou retornam com doença ativa após abandono, reforçado pelo etambutol $(E)$ por seis meses, na recomendação ministerial de 1995 (E-1R). Um segundo esquema de reserva, o Esquema-3 (E-3), com estreptomicina (S) e Z por 3 meses e E e etionamida (Et) por doze, é reservado para pacientes com falência ao tratamento inicial ou ao retratamento ${ }^{9}$. O regime de uso dos medicamentos recomendado, até 1998 , foi o de tomada diária e autoadministrado.

A cultura e o teste de sensibilidade (TS) não são realizados rotineiramente no país, o que dificulta a identificação do perfil da resistência inicial ou primária, A falência que determina a indicação dos esquemas terapêuticos, tem por base os achados da baciloscopia direta e, na sua ausência, os achados clínicos e radiológicos ${ }^{9}$. Desse modo, o conceito de TBMR no país, compreende formas da doença provocadas por cepas bacilares além de resistentes à $\mathrm{R}$ e $\mathrm{H}$, resistente a mais uma das drogas normatizadas (conceito bacteriológico) ou com falência ou impossibilidade de uso do E-3 (conceito operacional) ${ }^{182021}$.

Tal como a própria doença, a forma MR, não se apresenta por igual nas diversas regiões do mundo, variando conforme a evolução, o momento epidemiológico e a qualidade do controle da doença ${ }^{1528} 30$. Assim, o conhecimento das características e peculiaridades dos pacientes com TBMR em uma determinada região, constitui uma base importante para a elaboração de medidas de controle e propostas terapêuticas.

O presente estudo tem com objetivo identificar e avaliar algumas características da TBMR em um Serviço de Referência que possam contribuir na orientação de propostas terapêuticas e na política de controle do problema no país.

\section{MATERIAL E MÉTODOS}

Estudo de uma coorte de 4 anos (de 1995 a 1998), de pacientes diagnosticados como portadores de TBMR pulmonar, matriculados no Instituto Clemente Ferreira (ICF), Serviço de Referência da Secretaria de Estado da Saúde, especializado na atenção a este tipo de doente, na cidade de São Paulo.

Os critérios de inclusão foram: 1) diagnóstico confirmado pela recuperação do $M$. tuberculosis no escarro, tipificados pelos métodos tradicionais ; 2) resistência primária ou pós-primária à $\mathrm{R}, \mathrm{H}$ e mais uma terceira droga normatizada pelo MS ou resistência à $R$, $\mathrm{H}$ e resultados desfavoráveis ao E-3, falência bacteriológica ou impossibilidade de uso por intolerância medicamentosa; 3) informações sobre antecedentes terapêuticos suficientes para classificação do tipo de resistência.

Variáveis estudadas. Foram estudadas as seguintes variáveis: 1) sexo e idade; 2) tipos de MR com base nos antecedentes terapêuticos; 3 ) antecedentes de contágio; 4) fatores associados à multirresistência; 5) perfil de resistência às drogas usuais estabelecido pelo método das proporções de Canetti; 6) distribuição das lesões no RX de tórax convencional.

\section{RESULTADOS}

Foram estudados 182 pacientes, selecionados pelos critérios de inclusão no estudo, de 220 matriculados no ICF no período de 1995 a 1998 e considerados como portadores de TBMR. Excluídos 38, 22 não tipificados, 8 por não apresentarem um padrão de resistência compatível e 8 por informações sobre a doença insuficientes para os objetivos propostos para o estudo.

Cento e doze $(61,5 \%)$ do sexo masculino e 70 (38,5\%) do feminino, com uma idade que variou entre 16 e 64 , média de $35,7 \pm 6,8,73 \%$ entre 20 e 50 anos (Tabela 1 ).

Considerando a história de uso e evolução de tratamentos anteriores, os pacientes foram classificados com o seguinte perfil de MR: 1) MR primária englobando pacientes sem tratamento anterior e com teste de sensibilidade (TS) realizado durante 0 tratamento inicial de TB, com 11 (6\%) pacientes. 2) MR pós-primária - caracterizados pelo uso de tratamentos anteriores, com 134 (74\%) pacientes. De acordo com a evolução do tratamento anterior estes pacientes foram subdivididos em 81 (45\%) por interrupção devido ao abandono ou seguidos períodos de irregularidade; $42(23 \%)$ com recidiva após cura do E-1 e falência tanto ao retratamento como ao E-3 e 11 (6\%) por interrupção marcadamente relacionado à reações adversas. Alguns pacientes, apresentavam mais de um tipo de evolução, 
privilegiando-se na classificação a interrupção do tratamento ou abandono. Irregularidade persistente, com períodos somados com mais de 60 dias sem uso da medicação, foi considerada como interrupção, semelhante ao abandono. Neste grupo, a maioria não realizou ou não tinha informações sobre TS no momento do primeiro diagnóstico da TB, recuperadas em apenas 11 destes, sendo 8 sensíveis às drogas normatizadas

Tabela 1 - Sexo e idade em 182 pacientes com TBMR pulmonar do ICF $(S P)$ - 1995 a 1998.

\begin{tabular}{|c|c|c|c|c|c|}
\hline \multicolumn{2}{|c|}{ Homens } & \multicolumn{2}{|c|}{ Mulheres } & \multicolumn{2}{|c|}{ Total } \\
\hline $\mathrm{n}^{\circ}$ & $\%$ & $\mathrm{n}^{\circ}$. & $\%$ & $\mathrm{n}^{\circ}$. & $\%$ \\
\hline 112 & 61,5 & 70 & 38,5 & 182 & 100 \\
\hline \multicolumn{6}{|c|}{ Idade } \\
\hline \multicolumn{2}{|c|}{$<20=14(8 \%)$} & \multicolumn{2}{|c|}{20 a $50=133(73 \%)$} & \multicolumn{2}{|c|}{$>50=35(19 \%)$} \\
\hline
\end{tabular}

Variando entre 16 e 64 anos - média $=35,7+6,8$ e 3 apresentando resistência inicial a uma droga (2 à $\mathrm{He}$ 1 à S). 3) $M R$ indeterminada - identificados pela falência seqüencial aos E-1 e E-3 e informação de regularidade no uso dos medicamentos, com 37 (20\%) pacientes. Em apenas 2 pacientes foram recuperados o TS quando do diagnóstico inicial, ambos sem resistência às drogas normatizadas. A informação de regularidade foi fornecida pelos pacientes, sem nenhum tipo de supervisão durante os tratamentos realizados (Tabela 2).

Investigação sobre contágio foi documentada em 170 pacientes, 129 com contágio desconhecido e 41 (29\%) identificados, sendo 34/41 (83\%) intradomiciliares, $15 \mathrm{com}$ o caso índex ou foco não considerado MR e 19 com foco com TBMR. Entre os últimos, 8 classificados como MR primária, 7 como MR pós-primária (6 por abandono e 1 por reação adversa) e 4 como MR indeterminada. Contágio institucional para TBMR foi observado em 7/41 (17\%), com

Tabela 2 - Tipos de multirresistência e antecedentes terapêuticos em 182 pacientes com TBMR pulmonar do ICF $(S P)$ - 1995 a 1998.

\begin{tabular}{|c|c|c|c|c|c|}
\hline \multirow[t]{2}{*}{ Tipos de multirresistência } & \multirow[t]{2}{*}{ Antecedentes terapêuticos } & \multicolumn{2}{|c|}{ Subtotal } & \multicolumn{2}{|c|}{ Total } \\
\hline & & $\mathrm{n}^{\circ}$ & $\%$ & $\mathrm{n}^{\circ}$ & $\%$ \\
\hline \multirow[t]{2}{*}{ Primária } & contatos de TBMR & 8 & 4,0 & 11 & 6,0 \\
\hline & profissionais de saúde & 3 & 2,0 & & \\
\hline \multirow[t]{4}{*}{ Pós-primária } & abandono/irregularidade & 81 & 45,0 & & \\
\hline & recidiva do $E-1$ + falência com & 42 & 23,0 & 134 & 74,0 \\
\hline & retratamento do $\mathrm{E}-1$ e do $\mathrm{E}-3$ & & & & \\
\hline & reações adversas às drogas & 11 & 6,0 & & \\
\hline Indeterminada & informando o uso anterior regular das drogas & & & 37 & 20,0 \\
\hline Total & & & & 182 & 100,0 \\
\hline
\end{tabular}

5 profissionais de saúde, 1 carcereiro e 1 advogado criminalista com freqüência constante em presídios ${ }^{(*)}$, classificados como MR primária comprovada, 3 pacientes e 4, como MR pós-primária (3 por abandono e 1 por interrupção de tratamento anterior devido a reações adversas, HIV positivo). O contágio com TBMR alcançou $15 \%$ (26/170) dos casos estudados, sendo 19 domiciliares e 7 institucionais, com 11 (42\%) portadores de MR primária, 11 (42\%) pós-primária e 4 (16\%) indeterminada.

Delimitados 4 surtos familiares (3 ou mais indivíduos), com 3, 3, 4 e 6 em cada, envolvendo um total de 16 pacientes, 9 deles estudados na presente coorte onde não foi observado nenhum surto institucional. Nestes surtos, 8 tinham MR primária, 2 MR pós-primária (por abandono), 2 MR indeterminada e 4 sem informações (Tabela 3).

Entre os fatores associados à MR, o de maior freqüência foi o abandono e a irregularidade em tratamentos anteriores (81/182-45\%), seguido do etilismo (49/182-27\%); falência seqüencial aos esquemas de retratamento (42/182-23\%); contágio com TBMR (26/10$15 \%$ ) e reações adversas às drogas (11/182-6\%). Embora proposta como rotina, a sorologia anti-HIV não foi realizada em 6 pacientes, que se recusaram a realizar o teste e negavam riscos para esta infecção viral. Sorologia positiva para o HIV foi encontrada em 6 de 176 pacientes $(4 \%)$, classificados como MR pósprimária, 4 por abandono e 2 por reações adversas às drogas, um deles referindo contágio institucional e apresentando também irregularidade por efeitos adversos. Diabetes mellitus foi diagnosticada em outros 6 pacientes, metade com MR indeterminada e metade com MR pós primária (por abandono), e, 2 pacientes relataram gastrectomia anterior a TB, ambos do grupo de MR indeterminada. $\mathrm{Na}$ Tabela 4, são apresentados por ordem de freqüência os diversos fatores relacionados MR observados nesta série.

Quanto ao perfil de sensibilidade, todos os 182 eram resistentes à $\mathrm{R}$ e à $\mathrm{H}, 151$ (83\%) à S e 86 (47\%) ao $\mathrm{E}$. Foram desconsideradas as sensibilidades à $\mathrm{Z}$ e à Et, por não serem realizadas rotineiramente no ICF (Figura 1).

As lesões na radiologia convencional apresentaram distribuição unilateral em 28 (15\%) e bilateral em 154 (85\%). Todos os pacientes com lesões cavitárias, únicas ou múltiplas, apenas 35 (19\%) localizadas unilateralmente (Tabela 5).

\footnotetext{
*Todos os contatos institucionais foram considerados como contágio para TBMR.
} 
Tabela 3 - Antecedentes de contágio e surtos em 170 pacientes com TBMR pulmonar do ICF (SP) - 1995 a $1996^{1}$.

\begin{tabular}{lrrrr}
\hline Contágio & \multicolumn{4}{c}{ Pacientes } \\
\cline { 2 - 5 } & $\mathrm{n}^{\circ}$ & $\%$ & $\%$ & $\%$ \\
\hline Não identificado & 129 & 71,0 & \\
identificado & 41 & 29,0 & 100,0 & \\
$\quad$ intradomiciliar & 34 & & 83,0 & 100,0 \\
$\quad$ foco (TB não MR) ${ }^{2}$ & 15 & & & 37,0 \\
$\quad$ foco (TBMR) $^{3}$ & 19 & & & 63,0 \\
institucional $^{4}$ & 7 & 17,0 & \\
\hline
\end{tabular}

Surtos de TBMR: familiares $=\operatorname{com} 3,3,4$ e 6 casos $^{5}$ institucional $=$ nenhum observado nesta série

1. Em 12 casos não foi possível recuperar a história de contágio.

2. Nove com MR pós-primária (abandono) e 6 com indeterminada.

3. Oito com MR primária, 7 com pós-primária (6 abandono e 1 reação adversa), 4 com indeterminada.

4. MR primária em 3 e pós primária em 4 ( 3 por abandono e 1 reação adversa HIV positivo). Todos considerados contágio com TBMR. Contágio total com TBMR $=19+7=26 / 170(15 \%)$

5. Dos 16 casos ( 9 deles incluídos no presente estudo), 8 com MR primária, 2 com pósprimária (abandono), 2 com indeterminada e 4 sem informação.

Tabela 4 - Condições associadas à MR em 182 pacientes com TBMR pulmonar do ICF $(S P)$ - 1995 a 1998

\begin{tabular}{|c|c|c|}
\hline Fatores & $\mathrm{N}^{\circ}$ & $\%$ \\
\hline Abandono/Irregularidade $(\mathrm{N}=182)$ & 81 & 45 \\
\hline Etilismo $(\mathrm{N}=182)^{1}$ & 49 & 27 \\
\hline Falência aos esquemas de retratamento $(\mathrm{N}=182)$ & 42 & 23 \\
\hline Contágio com MR (N=170) & 26 & 15 \\
\hline Reações adversas às drogas $(\mathrm{N}=182)^{2}$ & 11 & 6 \\
\hline Co-infecção TB - HIV/AIDS $(\mathrm{N}=176)^{3}$ & 6 & 3 \\
\hline Diabetes mellitus $(\mathrm{N}=182)^{4}$ & 6 & 3 \\
\hline Gastrectomia $(\mathrm{N}=182)^{5}$ & 2 & 1 \\
\hline
\end{tabular}

1. Quarenta e quatro relacionados ao abandono (MR pós-primária) e 5 com MR indeterminada

2. Considerados como MR pós-primária, entre estes dois HIV positivos.

3. Todos com MR pós primária (4 por abandono e 2 relacionados a reações adversas.

4. MR indeterminada em 3 e 3 com MR pós-primária (por abandono).

5. Os dois no grupo de MR indeterminada.

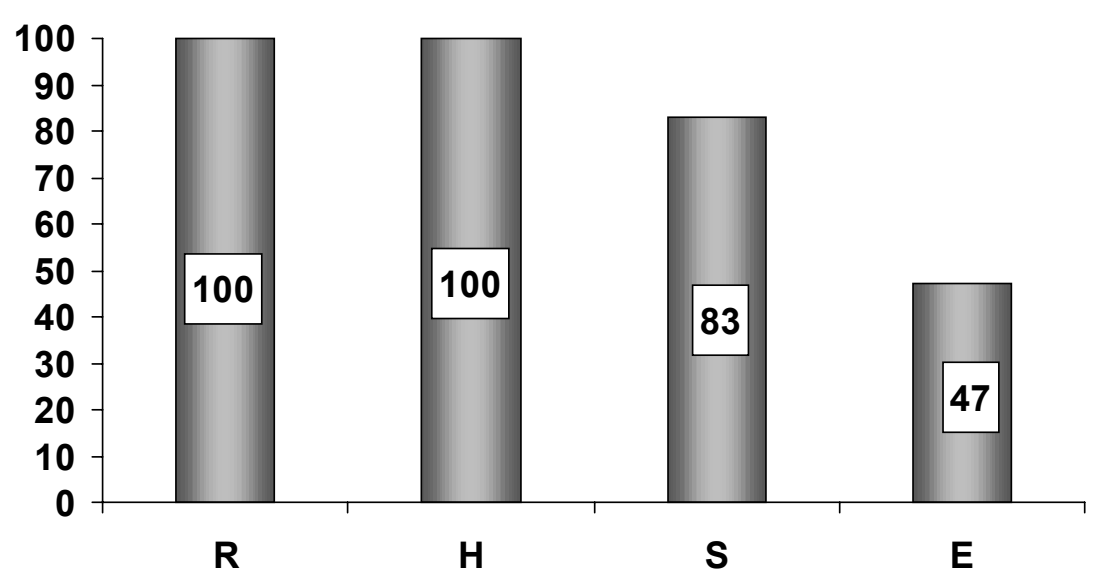

Figura 1 - Perfil da taxa de resistência às principais drogas usuais de 182 pacientes com TBMR pulmonar do ICF $(S P)-1995$ a 1998. 
Tabela 5 - Distribuição das lesões pulmonares e das cavidades na radiologia convencional em 182 pacientes com TBRM pulmonar do ICF (SP) - 1995 a 1998.

\begin{tabular}{lrrrrr}
\hline Distribuição & \multicolumn{2}{c}{ Lesões radiológicas } & & \multicolumn{2}{c}{ Cavidades $^{1}$} \\
\cline { 2 - 3 } \cline { 5 - 6 } & $\mathrm{n}^{\circ}$ & $\%$ & & $\mathrm{n}^{\circ}$ & $\%$ \\
\hline Unilaterais & 28 & 15,0 & & 35 & 19,0 \\
Bilaterais & 154 & 85,0 & & 147 & 81,0 \\
\hline Total & 182 & 100,0 & & 182 & 100,0 \\
\hline
\end{tabular}

1. Todos os pacientes com lesões cavitárias.

\section{DISCUSSÃO}

Desde 1992, quando dos primeiros surtos institucionais de TBMR nos Estados Unidos da América, que vem sendo destacado diferenças nas características desta forma da doença em nosso país em relação às observadas naqueles relatos ${ }^{15} 1830$.

Nos países que conquistaram um efetivo controle da TB, com o desenvolvimento social e uma ampla cobertura da quimioterapia, foram selecionadas cepas bacilares resistentes a $\mathrm{Se} \mathrm{H}$, medicamentos básicos então usados no tratamento, antes do advento da $\mathrm{R}$ nos meados da década de 60. Com o aumento da expectativa de vida, reativam-se casos de TB entre os idosos, facilitados pela decrescente imunidade da senescência e provocados exatamente por bacilos resistentes a $\mathrm{S} \mathrm{e} \mathrm{H}$, albergados em seus organismos. A incorporação da $R$, nestes países, não contou, portanto, com uma adequada proteção das desgastadas $\mathrm{HeS}$, o que justificaria, entre outras razões, o aumento crescente da resistência e MR acompanhada nas duas últimas décadas 152127282932 .

Ainda que a transferência de cepas MR por imigrantes também possa ser responsabilizada, a TBMR nos países desenvolvidos mantém uma clara relação com a coinfecção TB-HIV/AIDS e TB dos idosos. Apresenta-se nestes países, como surtos institucionais e localizados de MR primária e, embora constituam uma séria ameaça, são passíveis de controle pelos recursos econômicos e desenvolvimento técnico disponíveis ${ }^{1278}$.

Já nos países em desenvolvimento com industrialização e urbanização crescente, o Brasil entre eles, apresentaram uma epidemia tuberculosa historicamente retardada em relação aos países desenvolvidos. Muitos destes países, experimentaram uma importante queda da resistência, entre as décadas de 60 e 70, com a introdução de programas nacionais de controle da doença. Na década de 80, a incorporação da $\mathrm{R}$ em regimes de curta duração, constituiu um novo fator para o decréscimo da resistência para as drogas como $\mathrm{H}_{\text {e S}}{ }^{1632}$. Assim, a TBMR entre nós, revela características peculiares, próprias do desenvolvimento e controle da epidemia no país.

Desde a introdução de esquemas normatizados nacionais, para tratamento da TB, que vem se acumulando um resíduo de pacientes portadores de cepas bacilares com resistências múltiplas às drogas usuais, basicamente relacionado à interrupção do tratamento por abandono. Inicialmente com o Esquema standart que associava $\mathrm{S}, \mathrm{H}$ e ácido para-amino- salicílico (PAS), no início da década de 60; seguido por um curto período na década de 70 do esquema que introduziu a tiossemicarbazona (TSCZ) no lugar do PAS (não adotado nos Estados de São Paulo e Rio Grande do Sul). Tal resíduo e o uso de regimes autoadministrados, podem ter influenciado a opção por um esquema de reserva nas propostas de tratamento para a TB, quando da introdução do regime de curta duração com $R$, em 197917.

O esquema de curta duração estabelecido, apresentou uma alta eficácia, tanto para os casos novos como para os pacientes não curados ou recidivantes dos antigos esquemas, pois além da $R$, acresceu-se da $Z$, de reconhecido poder esterilizante ${ }^{102324}$. A parte dos significativos benefícios com a implantação de um esquema de alta eficácia e potência esterilizante, da redução do tempo de tratamento, do uso associado da $\mathrm{R}$ e da $\mathrm{H}$ em uma única cápsula, permaneciam inúmeros problemas relacionados a qualidade da rede de atenção à $T B$, com taxa de abandono médio de $12 \%$ nos primórdios da década de 80 , elevada para cerca de $14 \%$ no início da década de 90 , com números ainda maiores nas grandes capitais ${ }^{3} 5$. Mantinha-se constante a produção do resíduo de pacientes com cepas MR, de novo, no resíduo, a resistência múltipla à $\mathrm{R} \mathrm{e} \mathrm{H}$, principal dupla terapêutica atualmente conhecida, o que sem dúvida limita bastante as opções de tratamento ${ }^{10} 162329$.

Neste contexto, a MR no país, é um fenômeno anterior aos surtos institucionais relacionados a HIV/ AIDS que preocupa o primeiro mundo, com características próprias, cujo conhecimento, objetivo deste trabalho, podem ser úteis para o controle e orientação dos esquemas de tratamento.

A TBMR, nesta série, atingiu principalmente pacientes do sexo masculino $(61,5 \%)$, semelhante a outros estudos publicados no país ${ }^{4}{ }^{30}$ e a números relatados para tuberculosos sem tratamento anterior ${ }^{314}$. Predominaram os adultos jovens, a maioria (73\%) em faixa etária produtiva, entre 20 e 50 anos. A idade média de 35,7 foi semelhante à encontrada por Seiscento e cols ${ }^{30}$, de 37 anos, em 70 portadores de TBMR estudados no ICF, entre 1993 e 1994; por Dalcolmo e cols ${ }^{4}$, de 36,9 anos, em estudo multicêntrico nacional, com 197 doentes e Fortes $^{6}$ de 39,7, avaliando a falência do tratamento alternativo em 101 portadores de TBMR, com 71 curados e 30 falidos. 
Quanto ao tipo de MR, foi amplo o predomínio da MR pós-primária (74\%), principalmente relacionado ao abandono (45\%), seguido da baixa efetividade dos esquemas de retratamento (23\%). Números semelhantes foram encontrados por Seiscentos e cols ${ }^{30}$, com $80 \%$ de MR pós-primária, 54,3\% devido ao abandono e 14,3\% com recidiva após tratamentos anteriores. A MR pósprimária provocadas pelas reações adversas dos medicamentos de $6 \%$ neste estudo, foi de $11,4 \%$ naquele. A MR primária, melhor definida nesta série, foi de $6 \%$, sendo mal definida no trabalho de Seiscentos e cols ${ }^{30} \mathrm{e}$ de $8 \%$ no multicêntrico nacional de Dalcolmo e cols ${ }^{4}$. Este tipo MR deve ser mais numeroso, considerando que podem ser de primários, parte dos pacientes arrolados como portadores de MR indeterminada, resultante da não realização rotineira dos testes de sensibilidade no país, mas ainda assim, bem menos numerosos que os portadores de MR pós-primária.

Considerando um modelo de predomínio da MR primária, onde cepas bacilares únicas e sem influências da medicação, apresentam uma transmissão constante e mais intensa que cepas com MR pós-primária, cujo efeito protetor inicial das drogas diminui o tempo de transmissão, altera a biodiversidade das populações bacilares e interfere na comunicabilidade dos bacilos ${ }^{1927}$, é possível imaginar que o risco de uma grande epidemia de TBMR no Brasil é baixo. Colabora para esta hipótese a alta cobertura da vacinação BCG que, segundo Kritski e cols ${ }^{12}$, protegeria contatos intradomiciliares da transmissão de bacilos MR, além das observações de Melo e cols., no ICF, relatando que portadores de TBMR pós-primária, não curados com o tratamento alternativo, eliminaram cepas sensíveis no agravamento tardio da doença ${ }^{19}$, e que, filhos de MR pósprimários adoeceram, em sua maioria, com cepas sensíveis ou curaram quando tratados com drogas usuais $^{17}$.

Nos 26 pacientes que relataram antecedentes de contágio com TBMR, a transmissão institucional $(7 / 26=27 \%)$ foi quase 3 vezes inferior a intradomiciliar $(19 / 26=73 \%)$. Cerca de $42 \%$ (11/26) eram portadores de MR primária, sendo a maior ocorrência entre os contatos intradomiciliares $(8 / 11=72 \%)$. Diferentemente do que tem sido observado em países desenvolvidos, onde foram relatados surtos institucionais de MR primária relacionados a co-infecção TB-HIV/AIDS ${ }^{127}$. 0 estudo delimitou 6 surtos familiares, considerando um surto com pelo menos 3 casos de TBMR; e, nenhum institucional. Tais achados, são indicativos que os contatos intradomiciliares de MR, constitui um grupo importante no rastreamento e controle da TBMR, ainda mais se considerarmos serem tais surtos a principal fonte de MR primária definida na casuística. Estes números sugerem que, para os casos aqui revistos e provavelmente em todo o país, tal como a própria TB, a TBMR é basicamente social e não institucional.

Entre as condições e doenças associadas a TBMR nesta série, a de maior freqüência foi o abandono e a irregularidade com $45 \%$ (81/182), seguido do etilismo com $27 \%$ (49/182), da falência seqüencial aos esquemas de retratamento com $23 \%(42 / 182)$ e do contágio com portadores de cepas MR com 15\% (26/170). A interrupçâo por efeitos adversos com 11 (6\%), pode resultar da ausência ou de inadequadas orientações, ou ainda da baixa capacidade ou agilidade da rede de atenção a TB, em estabelecer condutas para solucionar problemas desta natureza. Foi pequena a influência da co-infecção TB-HV/ AIDS $(6 / 176=3 \%)$, semelhante aos achados de Fortes ${ }^{6}$ de $3 \%(3 / 101)$ e, o dobro do encontrado por Dalcolmo e cols ${ }^{4}$ com 1,6\% (3/187). Deve ser considerado, que os estudos foram realizados em unidades de referência para TB, o que possivelmente não dimensiona de forma adequada a participação da co-infecção TB-HIV-AIDS na TBMR. Tais percentuais, diferem bastante dos achados de Pinto ${ }^{26}$, que reviu a resistência às drogas antituberculosas, em 431 doentes de TB e HIV-positivo, matriculados num serviço de referência para HIV/AIDS. Esta autora, encontrou 82 pacientes com cepas resistentes, sendo $49(11,5 \%)$ com TBMR. Os tipos de resistências encontrados foram: MR primária em $13(26,5 \%)$ pacientes, pós-primária em 27 $(55,1 \%)$ e indeterminada em 9 (18,4\%). Provavelmente no país, a TBMR não apresenta ainda uma relação tão íntima com a co-infecção TB/HIV-AIDS, como acontece nos países desenvolvidos e nos africanos, porém, não parece ser tão baixa como encontrado na presente série. Outros fatores como dabetes mellitus (3\%) e gastrectomia prévia (1\%), relacionados a resposta imunológica deficitária foram poucos expressivos.

Quanto ao perfil de sensibilidade, mais da metade (53\%) dos pacientes ainda apresentavam sensibilidade ao $\mathrm{E}$, sendo ela, entre as drogas usuais, a de melhor aproveitamento nos esquemas alternativos testados no país ${ }^{4162330}$. Embora não tenha sido avaliado o real papel de sua inclusão nos ensaios, é preciso lembrar que o presente estudo, foi realizado antes da plena inclusão do $E$ em esquema de retratamento $(E-1 R)$ para recidivas e retorno com TB ativa após abandono ${ }^{9}$.

Considerando que $23 \%$ do total apresentavam TBMR por falência seqüencial ao retratamento com o $\mathrm{E}-1$ e E-3, é provável que o aporte do $\mathrm{E}$ como reforço no esquema $E-1 R$ com o objetivo de proteger a dupla $\mathrm{R}+\mathrm{H}$ e fazendo parte do $\mathrm{E}-3$ de reserva, tenha, como contrapartida, um aumento de sua resistência pósprimária, limitando seu uso em esquemas alternativos. Se isso for confirmado, há necessidade de uma urgente revisão, com vistas a formular um novo e mais potente esquema de reserva, agora com duas drogas já utilizadas anteriormente, o $\mathrm{E}$ a Z . A tendência seria a incorporação de uma fluoroquinolona no E-3. Embora dependendo de ensaios que comprovem uma maior eficácia e efetividade, esta incorporação praticamente transformaria o esquema de reserva num esquema para a TBMR, simplificando o procedimento e igualando nosso conceito de MR à literatura internacional, ou seja, definido apenas por seu aspecto bacteriológico. A S, ainda sensível em 31 (17\%) dos pacientes, dificilmente será reutilizada amplamente no tratamento da TBMR, 
pois além da baixa indicação, quando testada, mostrou resultados aquém da amicacina, como foi observado por Seiscento e cols ${ }^{30}$. Estas observações confirmam a necessidade de um esquema composto basicamente por drogas alternativas, diferentes das usuais, para o tratamento da TBMR no país.

Tendo como referência as recomendações do I Consenso Brasileiro de Tuberculose $(1997)^{18}$, indicando a cirurgia para casos de lesões cavitárias unilaterais, os achados de apenas 35 (19\%) pacientes com estas características na radiologia convencional, bem menor do que Fortes ${ }^{6}$, que encontrou 43/101 (42,6\%), parecem limitar a conduta cirúrgica a um grupo reduzido de portadores da TBMR, mais ainda, se a identificação das cavidades for realizada pela TC, um método reconhecidamente mais sensível. Os estudos publicados no país, entretanto, não foram suficientes, até o momento, para uma definição mais precisa da contribuição da cirurgia e mais ainda dos critérios seletivos para a indicação desta conduta ${ }^{11} 132530$.

Os achados desta série, com a compreensão de que representa uma casuística própria de um serviço de referência, onde de certa forma se concentram os portadores de TBMR, sugerem que:

1) O controle da TBMR pode ter como metas: a) redução da taxa de abandono, com especial atenção aos etilistas; b) reforço dos regimes de retratamento; $c$ ) tratamento dos pacientes portadores de TBMR; d) controle dos contatos intradomiciliares e institucionais; e) melhor orientação sobre procedimentos para efeitos adversos das drogas; e, f) avaliação mais acurada sobre o papel da co-infecção TB-HIV/AIDS na MR.

2) Para o tratamento das formas de TBMR: a) é fundamental o aporte de drogas alternativas nos esquemas; b) o $\mathrm{E}$, quando sensível, pode fazer parte destes esquemas; c) a conduta cirúrgica, beneficia apenas num número limitado de casos.

Estudos de TBMR em outros estados, e com séries mais amplas, podem colaborar para a universalização e estabelecimentos de prioridade das metas aqui relacionadas, além da indicação de outras não contempladas no presente trabalho.

\section{REFERÊNCIAS BIBLIOGRÁFICAS}

1. Brudney K, Dobkin J. Resurgent tuberculosis in New York City: human immunodeficiency virus, homelessness, and the decline of tuberculosis control programs. American Review of Respiratory Diseases 144:745-748, 1998.

2. Center for Disease Control and Prevention USA. National action plan to combat multidrug-resistant tuberculosis. Meeting the challenge of multidrug-resistant tuberculosis: summary of a conference. Management of persons exposed to multidrugresistant tuberculosis. MMWR 41 (RR-11), 1992.

3. Centro de Referência Prof. Hélio Fraga/Fundação Nacional de Saúde. Ministério da Saúde. Rio de Janeiro. Reunião de avaliação operacional e epidemiológica do PNCT na década de 80 . Documento Básico. Boletim de Pneumologia Sanitária. Rio de Janeiro. N Especial 1993.

4. Dalcolmo MP, Fortes A, Melo FAF, Motta R, Ide Neto J, Cardoso N, Andrade M, Barreto AW, Gerhardt G. Estudo de efetividade de esquemas alternativos para o tratamento da tuberculose multirresistente no Brasil. Jornal de Pneumologia 25:63-69, 1999.

5. Diniz LS, Gerhardt G, Miranda JÁ, Manceau JN. Efetividade do tratamento da tuberculose em oito municípios de capitais brasileiras. Boletim de Pneumologia Sanitária 3: 6-19, 1995.

6. Fortes AML. Tuberculose multirresistente: características de pacientes com falência de tratamento e fatores associados. Tese de Mestrado. Universidade Federal do Rio de Janeiro, 2000.

7. Frieden TR, Sterling T, Pablo-Mendes A, Kilbumm JD, Cauthen GM, Dooley SN. The emergence of drug-resistant-tuberculosis in New York City. New England Journal of Medicine 318:521-525, 1993.

8. Fujiwara PI, Frieden TR. Tuberculosis epidemiology and control in the inner-city In: Rom WN, Garay SM, Tuberculosis (eds) Little, Brown and Co. New York p.99-112, 1995.

9. Fundação Nacional de Saúde/CNPS. Manual de normas para o controle da tuberculose, $4^{a}$ Edição. Ministério da Saúde. Brasília, 1995.

10. Grosset JH. Bacteriological basis of short course chemotherapy for tuberculosis. Clinical in Chest Medicine 1:231-241, 1980.
11. Guimarães CA, Montessi J. Marsico GA, Perroni F, Mesquita E. A cirurgia da tuberculose pulmonar multirresistente. Jornal de Pneumologia 22(supl 1):S14-S15, 1996.

12. Kritski LA, Marques MJO, Rabahi MF, Vieira MAMS, WerneckBarroso E, Carvalho CE, Andrade GN, Barroso MJOR, Andrade LM, Gontijo PP, Riley LW. Transmission of tuberculosis to close contacts of patients with multidrug-resistant tuberculosis. American Journal of Respiratory and Critical Care Medicine 153:331-335, 1996.

13. Leite LPS, Costa ALP, Andrade RNS, Galvão T. Tratamento cirúrgico adjuvante de tuberculose pulmonar multirresistente. Jornal de Pneumologia 23:11-14, 1997.

14. Macedo LG, Afiune JB, Melo FAF. Características clínicas, radiológicas e bacteriológicas de pacientes portadores de tuberculose pulmonar. Jornal de Pneumologia 18 (supl. 1):118, 1992.

15. Melo FAF, Afiune JB. Drogas antituberculosas no Brasil: multirresistência. Ars Curandis 25:51-58, 1992.

16. Melo FAF, Afiune JB, Ribeiro LHG, Castelo A. Resistência primária do $M$. tuberculosis num serviço ambulatorial de referência em São Paulo: evolução por três décadas e comparação com outros estudos nacionais. Jornal de Pneumologia 22:3-7, 1996.

17. Melo FAF, Alves FM, Ogata IS, Seabra AS, Xavier MC, Savioli MTG. Adoecimento e evolução terapêutica de filhos de pacientes portadores de TBMR. Jornal de Pneumologia 26 (supl 3):S95, 2000.

18. Melo FAF, Dalcolmo MP, Ruffino Netto A (Editores/Revisores) I Consenso brasileiro de tuberculose - 1997. Jornal de Pneumologia 23:294-342, 1997.

19. Melo FAF, De Felice EAA, Spada DTA, Afiune JB. Influência das drogas na diversidade biológica das populações bacilíferas em pacientes portadores de tuberculose multirresistente (TBMR) e crônica (TBCR). Jornal de Pneumologia 26 (supl 3):S135, 2000.

20. Melo FAF, Ide Neto J, Seiscento M, Pinto JA, Afiune JB. Tuberculose multirresistente. Jornal de Pneumologia 19:73-82, 1993. 
21. Melo FAF, Seiscento M, Afiune JB, Ide Neto J, Noronha AMI. Tuberculose multirresistente no Brasil, uma definição operacional ou bacteriológica? Jornal de Pneumologia 22:1-2, 1996.

22. Melo FAF, Seiscento M. Ide Neto J, Noronha ANL, Cruz ML. A tuberculose multirresistente no Brasil In: Pneumologia: atualização e reciclagem - Sociedade Paulista de Pneumologia e Tisiologia, Editora Atheneu, São Paulo, vol II, p.239-243, 1997.

23. Natal S. Fatores de predição para a resistência aos tuberculostáticos. Tese de Doutorado. Instituto de Medicina Social da Universidade do Estado do Rio de Janeiro, 2000.

24. Penna ML, Hijjar MA, Feitosa JV, Teixeira GM, Gerhardt G, Neves JS. Resultados do tratamento de curta duração em doentes de tuberculose com recidiva. Jornal de Pneumologia 18 (supl):S139-140, 1982.

25. Pinto JAF, Kaemmerer A, Camargo JJP, Souza AAL, Picon PD, Rizzon CFC, Hoeffel Filho JR, Geyerr GRR. Ressecção pulmonar no tratamento da tuberculose In: Picon PD, Rizzon CFC, Ott WP (eds.) Tuberculose: epidemiologia, diagnóstico e tratamento em clínica e saúde pública. Medsi Editora Médica e Científica, Rio de Janeiro, p.589-600, 1993.
26. Pinto WP. Tuberculose e resistência a drogas em pacientes atendidos em um centro de referência para a sindrome da imunodeficiência adquirida em São Paulo, Brasil. Tese de Doutorado. Faculdade de Medicina da Universidade de São Paulo. 1998.

27. Rosemberg J. Tuberculose: Panorama global. Óbices para seu controle. Secretaria de Estado da Saúde do Ceará,1999.

28. Ruffino Netto A. Tuberculose MDR. Médicos 1:39-41, 1998.

29. Seiscento M, Melo FAF. Tuberculose multirresistente In:Veronesi R, Focaccia R (eds) Tratado de infectologia, Editora Atheneu. São Paulo, p. 954-957, 1996.

30. Seiscento M, Melo FAF, Ide Neto J, Noronha AML, Afiune JB, Inomata $\mathrm{T}$, Cruz ML. Tuberculose multirresistente (TBMR): aspectos clínico-laboratoriais, epidemiológicos e terapêuticos. Jornal de Pneumologia 23:237-244, 1997.

31. Simone PM, Dooley SW. The phenomenon of multidrug-resistant tuberculosis In: Rossman MD, MacGregor RR (eds) Tuberculosis: clinical management and new challenges. McGraw-Hill, Inc. New York. p.291-311, 1995.

32. Valderzis BP, Grosset J, Kantor I, Laszlo A. Drug-resistant tuberculosis laboratory issues. Tubercle Lung Diseases 75:1-7, 1994. 